\title{
On the fixed points of nonexpansive mappings in modular metric spaces
}

\author{
Afrah AN Abdou ${ }^{1 *}$ and Mohamed A Khamsi ${ }^{2,3}$
}

${ }^{\text {*Correspondence: }}$

aabdou@kau.edu.sa

${ }^{1}$ Department of Mathematics, King Abdulaziz University, P.O. Box 80203 ,

Jeddah, 21589, Saudi Arabia

Full list of author information is

available at the end of the article

\begin{abstract}
The notion of a modular metric on an arbitrary set and the corresponding modular spaces, generalizing classical modulars over linear spaces like Orlicz spaces, have been recently introduced. In this paper we investigate the existence of fixed points of modular nonexpansive mappings. We also discuss some compactness properties of the family of admissible sets in modular metric spaces with uniform normal structure property.

MSC: Primary 47H09; secondary 46B20; 47H10; 47E10
\end{abstract}

Keywords: nonexpansive mapping; $\Delta_{2}$-condition; fixed point; modular function space; modular metric spaces; Orlicz spaces

\section{Introduction}

The purpose of this paper is to give an outline of fixed point theory for nonexpansive mappings (i.e., mappings with the modular Lipschitz constant 1 ) on subsets of modular metric spaces which are natural generalization of classical modulars over linear spaces like Lebesgue, Orlicz, Musielak-Orlicz, Lorentz, Orlicz-Lorentz, Calderon-Lozanovskii spaces and many others. Modular metric spaces were introduced in $[1,2]$. The main idea behind this new concept is the physical interpretation of the modular. Informally speaking, whereas a metric on a set represents nonnegative finite distances between any two points of the set, a modular on a set attributes a nonnegative (possibly, infinite valued) 'field of (generalized) velocities': to each 'time' $\lambda>0$ (the absolute value of), an average velocity $\omega_{\lambda}(x, y)$ is associated in such a way that in order to cover the 'distance' between points $x, y \in X$, it takes time $\lambda$ to move from $\mathrm{x}$ to $\mathrm{y}$ with velocity $\omega_{\lambda}(x, y)$. But the way we approach the concept of modular metric spaces is different. Indeed, we look at these spaces as a nonlinear version of the classical modular spaces, introduced by Nakano [3], on vector spaces and modular function spaces, introduced by Musielak [4] and Orlicz [5].

In recent years, there was an increasing interest in the study of electrorheological fluids, sometimes referred to as 'smart fluids' (for instance, lithium polymetachrylate). For these fluids, modeling with sufficient accuracy using classical Lebesgue and Sobolev spaces, $L^{p}$ and $W^{1, p}$, where $p$ is a fixed constant, is not adequate, but rather the exponent $p$ should be able to vary $[6,7]$. One of the most interesting problems in this setting is the famous Dirichlet energy problem $[8,9]$. The classical technique used so far in studying this problem is converting the energy functional, naturally defined by a modular, to a convoluted and complicated problem which involves a norm (the Luxemburg norm). The modular metric approach is more natural and has not been used extensively.

(c) 2013 Abdou and Khamsi; licensee Springer. This is an Open Access article distributed under the terms of the Creative Commons Attribution License (http://creativecommons.org/licenses/by/2.0), which permits unrestricted use, distribution, and reproduction in any medium, provided the original work is properly cited. 
In many cases, particularly in applications to integral operators, approximation and fixed point results, modular-type conditions are much more natural as modular-type assumptions can be more easily verified than their metric or norm counterparts. In recent years, there has been a great interest in the study of the fixed point property in modular function spaces after the first paper [10] was published in 1990. More recently, the authors presented a fixed point result for pointwise nonexpansive and asymptotic pointwise nonexpansive acting in modular functions spaces [11]. The theory of nonexpansive mappings defined on convex subsets of Banach spaces has been well developed since the 1960s (see, e.g., Belluce and Kirk [12], Browder [13], Bruck [14], and Lim [15]) and generalized to other metric spaces (see, e.g., [16-18]) and modular function spaces (see, e.g., [10]). The corresponding fixed point results were then extended to larger classes of mappings like pointwise contractions and asymptotic pointwise contractions [18-22], and asymptotic pointwise nonexpansive mappings [11]. In [23], Penot presented an abstract version of Kirk's fixed point theorem [24] for nonexpansive mappings. Many results of a fixed point in metric spaces have been developed after Penot's formulation. Using Penot's work, the author in [25] proved some results in metric spaces with uniform normal structure similar to the ones known in Banach spaces.

In this paper we investigate the existence of fixed points of modular nonexpansive mappings defined in modular metric spaces. We also discuss some compactness properties of the family of admissible sets in modular metric spaces with uniform normal structure and prove similar results to the ones obtained in [25].

For more on metric fixed point theory and on modular function spaces, the reader may consult the books [26] and [27], respectively.

\section{Basic definitions and properties}

Let $\mathrm{X}$ be a nonempty set. Throughout this paper, for a function $\omega:(0, \infty) \times X \times X \rightarrow$ $[0, \infty]$, we write

$$
\omega_{\lambda}(x, y)=\omega(\lambda, x, y)
$$

for all $\lambda>0$ and $x, y \in X$.

Definition 2.1 [1,2] A function $\omega:(0, \infty) \times X \times X \rightarrow[0, \infty]$ is said to be a modular metric on $\mathrm{X}$ if it satisfies the following axioms:

(i) $x=y$ if and only if $\omega_{\lambda}(x, y)=0$ for all $\lambda>0$;

(ii) $\omega_{\lambda}(x, y)=\omega_{\lambda}(y, x)$ for all $\lambda>0$ and $x, y \in X$;

(iii) $\omega_{\lambda+\mu}(x, y) \leq \omega_{\lambda}(x, z)+\omega_{\mu}(z, y)$ for all $\lambda, \mu>0$ and $x, y, z \in X$.

If instead of (i) we have only the condition (i')

$$
\omega_{\lambda}(x, x)=0 \quad \text { for all } \lambda>0, x \in X,
$$

then $\omega$ is said to be a pseudomodular (metric) on $X$. A modular metric $\omega$ on $X$ is said to be regular if the following weaker version of (i) is satisfied:

$x=y \quad$ if and only if $\omega_{\lambda}(x, y)=0 \quad$ for some $\lambda>0$. 
Finally, $\omega$ is said to be convex if for $\lambda, \mu>0$ and $x, y, z \in X$, it satisfies the inequality

$$
\omega_{\lambda+\mu}(x, y) \leq \frac{\lambda}{\lambda+\mu} \omega_{\lambda}(x, z)+\frac{\mu}{\lambda+\mu} \omega_{\mu}(z, y) .
$$

Note that for a metric pseudomodular $\omega$ on a set $X$, and any $x, y \in X$, the function $\lambda \rightarrow$ $\omega_{\lambda}(x, y)$ is nonincreasing on $(0, \infty)$. Indeed, if $0<\mu<\lambda$, then

$$
\omega_{\lambda}(x, y) \leq \omega_{\lambda-\mu}(x, x)+\omega_{\mu}(x, y)=\omega_{\mu}(x, y) .
$$

Definition 2.2 $[1,2]$ Let $\omega$ be a pseudomodular on $X$. Fix $x_{0} \in X$. The two sets

$$
X_{\omega}=X_{\omega}\left(x_{0}\right)=\left\{x \in X: \omega_{\lambda}\left(x, x_{0}\right) \rightarrow 0 \text { as } \lambda \rightarrow \infty\right\}
$$

and

$$
X_{\omega}^{*}=X_{\omega}^{*}\left(x_{0}\right)=\left\{x \in X: \exists \lambda=\lambda(x)>0 \text { such that } \omega_{\lambda}\left(x, x_{0}\right)<\infty\right\}
$$

are said to be modular spaces (around $x_{0}$ ).

It is clear that $X_{\omega} \subset X_{\omega}^{*}$, but this inclusion may be proper in general. It follows from [1,2] that if $\omega$ is a modular on $X$, then the modular space $X_{\omega}$ can be equipped with a (nontrivial) metric generated by $\omega$ and given by

$$
d_{\omega}(x, y)=\inf \left\{\lambda>0: \omega_{\lambda}(x, y) \leq \lambda\right\}
$$

for any $x, y \in X_{\omega}$. If $\omega$ is a convex modular on $X$, according to [1,2] the two modular spaces coincide, i.e., $X_{\omega}^{*}=X_{\omega}$, and this common set can be endowed with the metric $d_{\omega}^{*}$ given by

$$
d_{\omega}^{*}(x, y)=\inf \left\{\lambda>0: \omega_{\lambda}(x, y) \leq 1\right\}
$$

for any $x, y \in X_{\omega}$. These distances are called Luxemburg distances (see example below for the justification).

Next we give the main example that motivated this paper.

Example 2.1 Let $X$ be a nonempty set and $\Sigma$ be a nontrivial $\sigma$-algebra of subsets of $X$. Let $\mathcal{P}$ be a $\delta$-ring of subsets of $X$ such that $E \cap A \in \mathcal{P}$ for any $E \in \mathcal{P}$ and $A \in \Sigma$. Let us assume that there exists an increasing sequence of sets $K_{n} \in \mathcal{P}$ such that $X=\bigcup K_{n}$. By $\mathcal{E}$ we denote the linear space of all simple functions with supports from $\mathcal{P}$. By $\mathcal{M}_{\infty}$ we denote the space of all extended measurable functions, i.e., all functions $f: X \rightarrow[-\infty, \infty]$ such that there exists a sequence $\left\{g_{n}\right\} \subset \mathcal{E},\left|g_{n}\right| \leq|f|$ and $g_{n}(x) \rightarrow f(x)$ for all $x \in X$. By $1_{A}$ we denote the characteristic function of the set $A$. Let $\rho: \mathcal{M}_{\infty} \rightarrow[0, \infty]$ be a nontrivial, convex and even function. We say that $\rho$ is a regular convex function pseudomodular if:

(i) $\rho(0)=0$;

(ii) $\rho$ is monotone, i.e., $|f(x)| \leq|g(x)|$ for all $x \in X$ implies $\rho(f) \leq \rho(g)$, where $f, g \in \mathcal{M}_{\infty}$;

(iii) $\rho$ is orthogonally subadditive, i.e., $\rho\left(f 1_{A \cup B}\right) \leq \rho\left(f 1_{A}\right)+\rho\left(f 1_{B}\right)$ for any $A, B \in \Sigma$ such that $A \cap B \neq \emptyset, f \in \mathcal{M}$; 
(iv) $\rho$ has the Fatou property, i.e., $\left|f_{n}(x)\right| \uparrow|f(x)|$ for all $x \in X$ implies $\rho\left(f_{n}\right) \uparrow \rho(f)$, where $f \in \mathcal{M}_{\infty}$;

(v) $\rho$ is order continuous in $\mathcal{E}$, i.e., $g_{n} \in \mathcal{E}$ and $\left|g_{n}(x)\right| \downarrow 0$ implies $\rho\left(g_{n}\right) \downarrow 0$.

Similarly, as in the case of measure spaces, we say that a set $A \in \Sigma$ is $\rho$-null if $\rho\left(g 1_{A}\right)=0$ for every $g \in \mathcal{E}$. We say that a property holds $\rho$-almost everywhere if the exceptional set is $\rho$-null. As usual we identify any pair of measurable sets whose symmetric difference is $\rho$-null as well as any pair of measurable functions differing only on a $\rho$-null set. With this in mind, we define

$$
\mathcal{M}(X, \Sigma, \mathcal{P}, \rho)=\left\{f \in \mathcal{M}_{\infty} ;|f(x)|<\infty \rho \text {-a.e. }\right\}
$$

where each $f \in \mathcal{M}(X, \Sigma, \mathcal{P}, \rho)$ is actually an equivalence class of functions equal $\rho$-a.e. rather than an individual function. Where no confusion exists, we write $\mathcal{M}$ instead of $\mathcal{M}(X, \Sigma, \mathcal{P}, \rho)$. Let $\rho$ be a regular function pseudomodular.

(a) We say that $\rho$ is a regular function semimodular if $\rho(\alpha f)=0$ for every $\alpha>0$ implies $f=0 \rho$-a.e.;

(b) We say that $\rho$ is a regular function modular if $\rho(f)=0$ implies $f=0 \rho$-a.e.

The class of all nonzero regular convex function modulars defined on $X$ is denoted by $\Re$. Let us denote $\rho(f, E)=\rho\left(f 1_{E}\right)$ for $f \in \mathcal{M}, E \in \Sigma$. It is easy to prove that $\rho(f, E)$ is a function pseudomodular in the sense of Def. 2.1.1 in [27] (more precisely, it is a function pseudomodular with the Fatou property). Therefore, we can use all results of the standard theory of modular function spaces as per the framework defined by Kozlowski in [27-29]; see also Musielak [4] for the basics of the general modular theory. Let $\rho$ be a convex function modular.

(a) The associated modular function space is the vector space $L_{\rho}(X, \Sigma)$, or briefly $L_{\rho}$ defined by

$$
L_{\rho}=\{f \in \mathcal{M} ; \rho(\lambda f) \rightarrow 0 \text { as } \lambda \rightarrow 0\} .
$$

(b) The following formula defines a norm in $L_{\rho}$ (frequently called the Luxemburg norm):

$$
\|f\|_{\rho}=\inf \{\alpha>0 ; \rho(f / \alpha) \leq 1\} .
$$

A modular function space furnishes a wonderful example of a modular metric space. Indeed, let $L_{\rho}$ be a modular function space. Define the function modular $\omega$ by

$$
\omega_{\lambda}(f, g)=\rho\left(\frac{f-g}{\lambda}\right)
$$

for all $\lambda>0$ and $f, g \in L_{\rho}$. Then $\omega$ is a modular metric on $L_{\rho}$. Note that $\omega$ is convex if and only if $\rho$ is convex. Moreover, we have

$$
\|f-g\|_{\rho}=d_{\omega}^{*}(f, g)
$$

for any $f, g \in L_{\rho}$.

Other easy examples may be found in $[1,2]$. 
Definition 2.3 Let $X_{\omega}$ be a modular metric space.

(1) The sequence $\left(x_{n}\right)_{n \in \mathbb{N}}$ in $X_{\omega}$ is said to be $\omega$-convergent to $x \in X_{\omega}$ if and only if $\omega_{1}\left(x_{n}, x\right) \rightarrow 0$ as $n \rightarrow \infty$. $x$ is called the $\omega$-limit of $\left(x_{n}\right)$.

(2) The sequence $\left(x_{n}\right)_{n \in N}$ in $X_{\omega}$ is said to be $\omega$-Cauchy if $\omega_{1}\left(x_{m}, x_{n}\right) \rightarrow 0$ as $m, n \rightarrow \infty$.

(3) A subset $M$ of $X_{\omega}$ is said to be $\omega$-closed if the $\omega$-limit of an $\omega$-convergent sequence of $M$ always belongs to $M$.

(4) A subset $M$ of $X_{\omega}$ is said to be $\omega$-complete if any $\omega$-Cauchy sequence in $M$ is an $\omega$-convergent sequence and its $\omega$-limit is in $M$.

(5) A subset $M$ of $X_{\omega}$ is said to be $\omega$-bounded if we have

$$
\delta_{\omega}(M)=\sup \left\{\omega_{1}(x, y) ; x, y \in M\right\}<\infty .
$$

In general, if $\lim _{n \rightarrow \infty} \omega_{\lambda}\left(x_{n}, x\right)=0$ for some $\lambda>0$, then we may not have $\lim _{n \rightarrow \infty} \omega_{\lambda}\left(x_{n}\right.$, $x)=0$ for all $\lambda>0$. Therefore, as it is done in modular function spaces, we say that $\omega$ satisfies $\Delta_{2}$-condition if this is the case, i.e., $\lim _{n \rightarrow \infty} \omega_{\lambda}\left(x_{n}, x\right)=0$ for some $\lambda>0$ implies $\lim _{n \rightarrow \infty} \omega_{\lambda}\left(x_{n}, x\right)=0$ for all $\lambda>0$. In [1] and [2], one can find a discussion about the connection between $\omega$-convergence and metric convergence with respect to the Luxemburg distances. In particular, we have

$$
\lim _{n \rightarrow \infty} d_{\omega}\left(x_{n}, x\right)=0 \quad \text { if and only if } \quad \lim _{n \rightarrow \infty} \omega_{\lambda}\left(x_{n}, x\right)=0 \quad \text { for all } \lambda>0
$$

for any $\left\{x_{n}\right\} \in X_{\omega}$ and $x \in X_{\omega}$. And in particular we have that $\omega$-convergence and $d_{\omega}$ convergence are equivalent if and only if the modular $\omega$ satisfies the $\Delta_{2}$-condition. Moreover, if the modular $\omega$ is convex, then we know that $d_{\omega}^{*}$ and $d_{\omega}$ are equivalent, which implies

$$
\lim _{n \rightarrow \infty} d_{\omega}^{*}\left(x_{n}, x\right)=0 \quad \text { if and only if } \quad \lim _{n \rightarrow \infty} \omega_{\lambda}\left(x_{n}, x\right)=0 \quad \text { for all } \lambda>0
$$

for any $\left\{x_{n}\right\} \in X_{\omega}$ and $x \in X_{\omega}[1,2]$. Another question that arises in this setting is the uniqueness of the $\omega$-limit. Assume that $\omega$ is regular, and let $\left\{x_{n}\right\} \in X_{\omega}$ be a sequence such that $\left\{x_{n}\right\} \omega$-converges to $a \in X_{\omega}$ and $b \in X_{\omega}$. Then we have

$$
\omega_{2}(a, b) \leq \omega_{1}\left(a, x_{n}\right)+\omega_{1}\left(x_{n}, b\right)
$$

for any $n \geq 1$. Our assumptions imply $\omega_{2}(a, b)=0$. Since $\omega$ is regular, we get $a=b$, i.e., the $\omega$-limit of a sequence is unique.

Let $(X, \omega)$ be a modular metric space. Throughout the rest of this work, we assume that $\omega$ satisfies the Fatou property, i.e., if $\left\{x_{n}\right\} \omega$-converges to $x$ and $\left\{y_{n}\right\} \omega$-converges to $y$, then we must have

$$
\omega_{1}(x, y) \leq \liminf _{n \rightarrow \infty} \omega_{1}\left(x_{n}, y_{n}\right)
$$

For any $x \in X_{\omega}$ and $r \geq 0$, we define the modular ball

$$
B_{\omega}(x, r)=\left\{y \in X_{\omega} ; \omega_{1}(x, y) \leq r\right\} .
$$

Note that if $\omega$ satisfies the Fatou property, then modular balls are $\omega$-closed. An admissible subset of $X_{\omega}$ is defined as an intersection of modular balls. Denote by $\mathcal{A}_{\omega}\left(X_{\omega}\right)$ the family 
of admissible subsets of $X_{\omega}$. Note that $\mathcal{A}_{\omega}\left(X_{\omega}\right)$ is stable by intersection. At this point we need to define the concept of Chebyshev center and radius in modular metric spaces. Let $A \subset X$ be a nonempty $\omega$-bounded subset. For any $x \in A$, define

$$
r_{x}(A)=\sup \left\{\omega_{1}(x, y) ; y \in A\right\} .
$$

The Chebyshev radius of $A$ is defined by

$$
R_{\omega}(A)=\inf \left\{r_{x}(A) ; x \in A\right\} .
$$

Obviously, we have $R_{\omega}(A) \leq r_{x}(A) \leq \delta_{\omega}(A)$ for any $x \in A$. The Chebyshev center of $A$ is defined as

$$
\mathcal{C}_{\omega}(A)=\left\{x \in A ; r_{x}(A)=R_{\omega}(A)\right\} .
$$

Throughout the remainder of this work, for a subset $A$ of a modular metric space $X_{\omega}$, set

$$
\operatorname{cov}_{\omega}(A)=\bigcap\{B: B \text { is a modular ball and } A \subset B\} .
$$

Recall that $A$ is $\omega$-bounded if $\delta_{\omega}(A)=\sup \left\{\omega_{1}(x, y) ; x, y \in A\right\}<\infty$.

Definition 2.4 Let $(X, \omega)$ be a modular metric space. Let $C$ be a nonempty subset of $X_{\omega}$.

(i) We say that $\mathcal{A}_{\omega}(C)$ is compact if any family $\left(A_{\alpha}\right)_{\alpha \in \Gamma}$ of elements of $\mathcal{A}_{\omega}(C)$ has a nonempty intersection provided $\bigcap_{\alpha \in F} A_{\alpha} \neq \emptyset$ for any finite subset $F \subset \Gamma$.

(ii) We say that $\mathcal{A}_{\omega}(C)$ is countably compact or satisfies the property $(R)$ if any sequence $\left(A_{n}\right)_{n \geq 1}$ of elements of $\mathcal{A}_{\omega}(C)$, which are nonempty and decreasing, has a nonempty intersection.

(iii) We say that $\mathcal{A}_{\omega}(C)$ is normal if for any $A \in \mathcal{A}_{\omega}(C)$, not reduced to one point, $\omega$-bounded, we have $R_{\omega}(A)<\delta_{\omega}(A)$.

(iv) We say that $\mathcal{A}_{\omega}(C)$ is uniformly normal if there exists $c \in(0,1)$ such that for any $A \in \mathcal{A}_{\omega}(C)$, not reduced to one point, $\omega$-bounded, we have $R_{\omega}(A) \leq c \delta_{\omega}(A)$.

Remark 2.1 Note that if $\mathcal{A}_{\omega}\left(X_{\omega}\right)$ is compact, then $X_{\omega}$ is $\omega$-complete. Indeed, let $\left\{x_{n}\right\} \subset X_{\omega}$ be an $\omega$-Cauchy sequence. Set

$$
r_{n}=\sup _{m, s \geq n} \omega_{1}\left(x_{m}, x_{s}\right)
$$

for any $n \geq 1$. Since $\left\{x_{n}\right\}$ is an $\omega$-Cauchy sequence, then $\lim _{n \rightarrow \infty} r_{n}=0$. By the definition of $r_{n}$, we get $x_{m} \in B_{\omega}\left(x_{n}, r_{n}\right)$ for any $n \geq 1$ and $m \geq n$. Hence, for any $n_{1}, n_{2}, \ldots, n_{p} \geq 1$, we have

$$
x_{m} \in \bigcap_{1 \leq i \leq p} B_{\omega}\left(x_{n_{i}}, r_{n_{i}}\right)
$$

for any $m \geq \max \left\{n_{1}, n_{2}, \ldots, n_{p}\right\}$. Since $\mathcal{A}_{\omega}\left(X_{\omega}\right)$ is compact, then

$$
C=\bigcap_{n \geq 1} B_{\omega}\left(x_{n}, r_{n}\right) \neq \emptyset .
$$


If $z \in C$, then we have $\omega_{1}\left(x_{n}, z\right) \leq r_{n}$ for any $n \geq 1$. Hence $\left\{x_{n}\right\} \omega$-converges to $z$, which completes the proof of our statement.

\section{Main results}

Let us first start this section with the definition of nonexpansive mappings in the modular metric sense.

Definition 3.1 Let $(X, \omega)$ be a modular metric space. Let $C$ be a nonempty subset of $X_{\omega}$. A mapping $T: C \rightarrow C$ is said to be $\omega$-nonexpansive if

$$
\omega_{1}(T(x), T(y)) \leq \omega_{1}(x, y) \quad \text { for any } x, y \in C .
$$

For such a mapping, we denote by $\operatorname{Fix}(T)$ the set of its fixed points, i.e., $\operatorname{Fix}(T)=\{x \in$ $C ; T(x)=x\}$.

In $[1,2]$ the author defined Lipschitzian mappings in modular metric spaces and proved some fixed point theorems. Our definition is more general. Indeed, in the case of modular function spaces, it is proved in [10] that

$$
\omega_{\lambda}(T(x), T(y)) \leq \omega_{\lambda}(x, y) \quad \text { for any } \lambda>0
$$

if and only if $d_{\omega}(T(x), T(y)) \leq d_{\omega}(x, y)$ for any $x, y \in C$. Next we give an example, which first appeared in [10], of a mapping which is $\omega$-nonexpansive in our sense but fails to be nonexpansive with respect to $d_{\omega}$.

Example 3.1 Let $X=(0, \infty)$. Define the Musielak-Orlicz function modular on the space of all Lebesgue measurable functions by

$$
\rho(f)=\frac{1}{e^{2}} \int_{0}^{\infty}|f(x)|^{x+1} d m(x) .
$$

Let $B$ be the set of all measurable functions $f:(0, \infty) \rightarrow \mathbb{R}$ such that $0 \leq f(x) \leq \frac{1}{2}$. Consider the map

$$
T(f)(x)= \begin{cases}f(x-1) & \text { for } x \geq 1 \\ 0 & \text { for } x \in[0,1]\end{cases}
$$

Clearly, $T(B) \subset B$. In [10], it was proved that for every $\lambda \leq 1$ and for all $f, g \in B$, we have

$$
\rho(\lambda(T(f)-T(g))) \leq \lambda \rho(\lambda(f-g)) .
$$

This inequality clearly implies that $T$ is $\omega$-nonexpansive. On the other hand, if we take $f=1_{[0,1]}$, then

$$
\|T(f)\|_{\rho}>e \geq\|f\|_{\rho},
$$

which clearly implies that $T$ is not $d_{\omega}$-nonexpansive.

Next we discuss the analog of Kirk's fixed point theorem [24] in modular metric spaces. 
Theorem 3.1 Let $(X, \omega)$ be a modular metric space. Let $C$ be a nonempty $\omega$-closed $\omega$-bounded subset of $X_{\omega}$. Assume that the family $\mathcal{A}_{\omega}(C)$ is normal and compact. Let $T: C \rightarrow C$ be $\omega$-nonexpansive. Then $T$ has a fixed point.

Proof Since $\mathcal{A}_{\omega}(C)$ is compact, there exists a minimal nonempty $A \in \mathcal{A}_{\omega}(C)$ such that $T(A) \subset A$. It is easy to check that $\operatorname{cov}_{\omega}(T(A))=A$. Let us prove that $\delta_{\omega}(A)=0$, i.e., $A$ is reduced to one point. Suppose that $\delta_{\omega}(A) \neq 0$. For any $x \in A$, set

$$
r_{x}(A)=\sup \left\{\omega_{1}(x, y) ; y \in A\right\} \leq \delta_{\omega}(A) \leq \delta_{\omega}(C)<\infty .
$$

Since $T$ is $\omega$-nonexpansive, we have $T(A) \subset B_{\omega}\left(T(x), r_{x}(A)\right)$ for any $x \in A$. Hence

$$
\operatorname{cov}_{\omega}(T(A)) \subset B_{\omega}\left(T(x), r_{x}(A)\right) .
$$

So, $r_{T(x)}(A) \leq r_{x}(A)$ for any $x \in A$. Next we fix $a \in A$ and define

$$
A_{a}=\left\{x \in A ; r_{x}(A) \leq r_{a}(A)\right\} .
$$

Clearly, $A_{a}$ is not empty since $a \in A_{a}$. Moreover, we have

$$
A_{a}=\bigcap_{x \in A} B_{\omega}\left(x, r_{a}(A)\right) \cap A \in \mathcal{A}_{\omega}(C) .
$$

And since $r_{T(x)}(A) \leq r_{x}(A)$, for any $x \in A$, we get $T\left(A_{a}\right) \subset A_{a}$. The minimality behavior of $A$ implies $A_{a}=A$. In particular we have $r_{x}(A)=r_{a}(A)$ for any $x \in A$. Hence $\delta_{\omega}(A)=$ $\sup _{x \in A} r_{x}(A)=r_{a}(A)$ for any $a \in A$. Since $\mathcal{A}_{\omega}(C)$ is normal, we get $\delta_{\omega}(A)<\delta_{\omega}(A)$, which is a contradiction. Thus we must have $\delta_{\omega}(A)=0$, i.e., $A$ is reduced to one point which is fixed by $T$.

Next we give a constructive result discovered by Kirk [30] which relaxes the compactness assumption in the above theorem. The main ingredient in Kirk's constructive proof is a technical lemma due to Gillespie and Williams [31]. The next lemma is a modular version of the Gillespie and Williams result.

Lemma 3.1 Let $(X, \omega)$ be a modular metric space, and let $C$ be a nonempty $\omega$-bounded subset of $X_{\omega}$. Let $T: C \rightarrow C$ be an $\omega$-nonexpansive mapping. Assume that $\mathcal{A}_{\omega}(C)$ is normal. Let $A \in \mathcal{A}_{\omega}(C)$ be nonempty and $T$-invariant, i.e., $T(A) \subset A$. Then there exists a nonempty $A_{0} \in \mathcal{A}_{\omega}(C)$, which is $T$-invariant, such that

$$
\delta_{\omega}\left(A_{0}\right) \leq \frac{\delta_{\omega}(A)+R_{\omega}(A)}{2} .
$$

Proof Set $r=\frac{1}{2}\left(\delta_{\omega}(A)+R_{\omega}(A)\right)$. We assume that $\delta_{\omega}(A)>0$, otherwise we can take the set $A=A_{0}$. Since $\mathcal{A}_{\omega}(C)$ is normal, we have $R_{\omega}(A)<\delta_{\omega}(A)$. Hence $R_{\omega}(A)<r$, which implies the existence of $a \in A$ such that $r_{a}(A)<r$. Therefore, the set

$$
D=\left\{a \in A: A \subset B_{\omega}(a, r)\right\}=\bigcap_{x \in A} B_{\omega}(x, r) \cap A
$$


is a nonempty admissible subset of $C$. Note that there is no reason for $D$ to be $T$-invariant. Consider the family

$$
\mathcal{F}=\left\{M \in \mathcal{A}_{\omega}(C): D \subset M \text { and } T(M) \subset M\right\} .
$$

Note that $\mathcal{F}$ is nonempty since $C \in \mathcal{F}$. Set $L=\bigcap_{M \in \mathcal{F}} M$. The set $L$ is an admissible subset of $C$ which contains $D$. Using the definition of $\mathcal{F}$, we deduce that $L$ is $T$-invariant. Consider $B=D \cup T(L)$ and observe that $\operatorname{cov}_{\omega}(B)=L$. Indeed, since $B \subset T(L) \subset L$ and $L \in \mathcal{A}_{\omega}(C)$, we have $\operatorname{cov}_{\omega}(B) \subset L$. From this we obtain

$$
T\left(\operatorname{cov}_{\omega}(B)\right) \subset T(L) \subset B \subset \operatorname{cov}_{\omega}(B) .
$$

Hence $\operatorname{cov}_{\omega}(B) \in \mathcal{F}$ and $L \subset \operatorname{cov}_{\omega}(B)$. This gives the desired equality. Define

$$
A_{0}=\left\{x \in L: L \subset B_{\omega}(x, r)\right\} .
$$

We claim that $A_{0}$ is the desired set. Observe that $A_{0}$ is nonempty since it contains $D$ (by the definition of $D$ ). Using the same argument, we can prove that $A_{0}$ is an admissible subset of $C$. On the other hand, it is clear that $\delta_{\omega}\left(A_{0}\right) \leq r$. To complete the proof, we have to show that $A_{0}$ is $T$-invariant. Let $x \in A_{0}$. By the definition of $A_{0}$, we have $L \subset B_{\omega}(x, r)$. Since $T$ is $\omega$-nonexpansive, we have

$$
T(L) \subset B_{\omega}(T(x), r) .
$$

For any $y \in D$, there holds $L \subset B_{\omega}(y, r)$. But $T(x) \in L$, so $T(x) \in B_{\omega}(y, r)$, which implies $y \in B_{\omega}(T(x), r)$. Hence $D \subset B_{\omega}(T(x), r)$ holds. Since $B=D \cup T(L)$, we get $B \subset B_{\omega}(T(x), r)$. Therefore, we must have

$$
\operatorname{cov}_{\omega}(B)=L \subset B_{\omega}(T(x), r) .
$$

By the definition of $A_{0}$, it follows that $T(x) \in A_{0}$. In other words, $A_{0}$ is $T$-invariant. Let $x, y \in A_{0}$, then $x, y \in L$, which implies $\omega_{1}(x, y) \leq r_{x}(L) \leq r$, i.e.,

$$
\delta_{\omega}\left(A_{0}\right) \leq r=\frac{\delta_{\omega}(A)+R_{\omega}(D)}{2} .
$$

Next we give the analogue of the main fixed point result in [30].

Theorem 3.2 Let $(X, \omega)$ be an $\omega$-complete modular metric space, and let $C$ be a nonempty $\omega$-closed $\omega$-bounded subset of $X_{\omega}$. Assume that the family $\mathcal{A}_{\omega}(C)$ is uniformly normal and $T: C \rightarrow C$ is $\omega$-nonexpansive. Then $T$ has a fixed point.

Proof Since $C$ is $\omega$-bounded, we have $C \in \mathcal{A}_{\omega}(C)$ since

$$
C=B_{\omega}\left(x, \delta_{\omega}(C)\right) \cap C \quad \text { for any } x \in C \text {. }
$$


Now, let us take $C=A_{0}$ and $T(C) \subset C$. Since $\mathcal{A}_{\omega}(C)$ is uniformly normal, there exists $c \in(0,1)$ such that

$$
R_{\omega}(A) \leq c \delta_{\omega}(A) \quad \text { for any } A \in \mathcal{A}_{\omega}(C) .
$$

By Lemma 3.1, there exists $A_{1} \in \mathcal{A}_{\omega}(C)$ such that $A_{1} \subset A_{0}, T\left(A_{1}\right) \subset A_{1}$ and satisfies

$$
\delta_{\omega}\left(A_{1}\right) \leq \frac{R\left(A_{0}\right)+\delta_{\omega}\left(A_{0}\right)}{2} .
$$

Using the induction argument, we build a sequence $\left\{A_{n}\right\} \subset \mathcal{A}_{\omega}(C)$ such that $A_{n+1} \subset A_{n}$, $T\left(A_{n+1}\right) \subset A_{n+1}$ and

$$
\delta_{\omega}\left(A_{n+1}\right) \leq \frac{R\left(A_{n}\right)+\delta_{\omega}\left(A_{n}\right)}{2} .
$$

Since $R_{\omega}\left(A_{n}\right) \leq c \delta_{\omega}\left(A_{n}\right)$, we get

$$
\delta_{\omega}\left(A_{n+1}\right) \leq\left(\frac{1+c}{2}\right) \delta_{\omega}\left(A_{n}\right)
$$

which implies that

$$
\delta_{\omega}\left(A_{n}\right) \leq\left(\frac{1+c}{2}\right)^{n} \delta_{\omega}(C) .
$$

Now, since $\delta_{\omega}(C)<\infty$, we get

$$
\lim _{n \rightarrow \infty} \delta_{\omega}\left(A_{n}\right)=0
$$

Let $x_{n} \in A_{n}$ for any $n \geq 1$. Then $\left\{x_{n}\right\}$ is an $\omega$-Cauchy sequence. Since $X_{\omega}$ is $\omega$-complete and $C$ is an $\omega$-closed subset of $X_{\omega}$, then $C$ is $\omega$-complete. Thus $\left\{x_{n}\right\} \omega$-converges to $x \in C$. Since $A_{n+1} \subset A_{n}$ and $A_{n}$ is $\omega$-closed for any $n \geq 1$, then $x \in A_{n}$ for any $n \geq 1$. Thus $\bigcap_{n} A_{n}$ is not empty and clearly is reduced to the single point $x$. Indeed, let $y \in \bigcap_{n} A_{n}$, then $y \in A_{n}$ for any $n \geq 1$. Hence

$$
\omega_{1}(x, y) \leq \delta_{\omega}\left(A_{n}\right) .
$$

Since $\lim _{n \rightarrow \infty} \delta_{\omega}\left(A_{n}\right)=0$, we get $\omega_{1}(x, y)=0$. Since $\omega$ is regular, we get $y=x$, i.e., $\bigcap_{n} A_{n}=\{x\}$. Since $T\left(A_{n}\right) \subset A_{n}$ for any $n \geq 1$, we get $T(x)=x$.

The following technical proposition is needed to show an analogue to the main result in [25].

Proposition 3.1 Let $(X, \omega)$ be an $\omega$-complete modular metric space, and let $C$ be a nonempty $\omega$-closed $\omega$-bounded subset of $X_{\omega}$. Assume that the family $\mathcal{A}_{\omega}(C)$ is uniformly normal. Consider the Cartesian product $C_{\infty}=\prod_{n \geq 1}$ C. Define $\Omega:(0, \infty) \times C \times C \rightarrow[0, \infty]$ by

$$
\Omega_{\lambda}\left(\left(x_{n}\right),\left(y_{n}\right)\right)=\sup _{n \geq 1} \omega_{\lambda}\left(x_{n}, y_{n}\right) .
$$


Then:

(i) $\left(C_{\infty}, \Omega\right)$ is an $\Omega$-complete modular metric space.

(ii) $C_{\infty}$ is $\Omega$-bounded with $\delta_{\Omega}\left(C_{\infty}\right)=\delta_{\omega}(C)<\infty$.

(iii) For any $\left(x_{n}\right) \in C_{\infty}$ and $r \in[0, \infty]$, we have

$$
B_{\Omega}\left(\left(x_{n}\right), r\right)=\prod_{n \geq 1} B_{\omega}\left(x_{n}, r\right)
$$

where

$$
B_{\Omega}\left(\left(x_{n}\right), r\right)=\left\{\left(y_{n}\right) \in C_{\infty} ; \Omega_{1}\left(\left(x_{n}\right),\left(y_{n}\right)\right) \leq r\right\}
$$

and $B_{\omega}\left(x_{n}, r\right)=\left\{y \in C ; \omega_{1}\left(x_{n}, y\right) \leq r\right\}$. This implies that for any $A \in \mathcal{A}_{\Omega}\left(C_{\infty}\right)$, we have $A=\prod_{n \geq 1} A_{n}$, where $A_{n} \in \mathcal{A}_{\Omega}(C)$.

(iv) $\mathcal{A}_{\Omega}\left(C_{\infty}\right)$ is uniformly normal.

Proof The proofs of (i), (ii), (iii) are easy and left to the reader. Let us prove (iv). Indeed, let $A \in \mathcal{A}_{\omega}\left(C_{\infty}\right)$ be nonempty and not reduced to one point. Then (iii) implies that $A=$ $\prod_{n \geq 1} A_{n}$, where $A_{n} \in \mathcal{A}_{\omega}(C)$. Let $\varepsilon>0$. Since $\mathcal{A}_{\omega}(C)$ is uniformly normal, there exists $c \in$ $[0,1)$ such that for any $n \geq 1$, for which $A_{n}$ is not reduced to one point, there exists $x_{n} \in A_{n}$ such that

$$
r_{x_{n}}\left(A_{n}\right) \leq(c+\varepsilon) \delta_{\omega}\left(A_{n}\right)
$$

Hence

$$
r_{\left(x_{n}\right)}(A)=\sup _{\left(y_{n}\right) \in A} \Omega\left(\left(x_{n}\right),\left(y_{n}\right)\right)=\sup _{\left(y_{n}\right) \in A}\left(\sup _{n \geq 1} \omega_{1}\left(x_{n}, y_{n}\right)\right),
$$

which implies

$$
r_{\left(x_{n}\right)}(A) \leq(c+\varepsilon) \sup _{n \geq 1} \delta_{\omega}\left(A_{n}\right)=(c+\varepsilon) \delta_{\Omega}(A) .
$$

So, $R_{\Omega}(A) \leq(c+\varepsilon) \delta_{\Omega}(A)$. Since $\varepsilon$ was arbitrary, we get $R_{\Omega}(A) \leq c \delta_{\Omega}(A)$. This completes the proof of (iv).

The following theorem shows that although we do not need compactness of the family of admissible sets in Theorem 3.2, its assumptions imply a weaker form of compactness, mainly countable compactness.

Theorem 3.3 Let $(X, \omega)$ be an $\omega$-complete modular metric space, and let $C$ be a nonempty $\omega$-closed $\omega$-bounded subset of $X_{\omega}$. Assume that the family $\mathcal{A}_{\omega}(C)$ is uniformly normal. Then $\mathcal{A}_{\omega}(C)$ has the property $(R)$.

Proof Let $\left\{A_{n}\right\}$ be a decreasing sequence of nonempty subsets of $C$, with $A_{n} \in \mathcal{A}_{\omega}(C)$. Consider the modular metric space $\left(C_{\infty}, \Omega\right)$ defined in Proposition 3.1. Set $A=\prod_{n} A_{n}$. 
Then $A \in \mathcal{A}_{\Omega}\left(C_{\infty}\right)$. Since $\mathcal{A}_{\Omega}\left(C_{\infty}\right)$ is uniformly normal, then $\mathcal{A}_{\Omega}(A)$ is uniformly normal. Consider the shift $T: A \rightarrow A$ defined by

$$
T\left(\left(x_{n}\right)\right)=\left(x_{n+1}\right) .
$$

Obviously, $T$ is $\omega$-nonexpansive. Theorem 3.2 implies that $T$ has a fixed point, i.e., there exists $\left(x_{n}\right) \in A$ such that $T\left(\left(x_{n}\right)\right)=\left(x_{n}\right)$. The definition of $T$ forces $\left\{x_{n}\right\}$ to be a constant sequence, i.e., $x_{n}=x$, for any $n \geq 1$. Obviously, we have $x \in A_{n}$ for any $n \geq 1$, which implies $\bigcap_{n \geq 1} A_{n} \neq \emptyset$.

\section{Competing interests}

The authors declare that they have no competing interests.

\section{Authors' contributions}

All authors participated in the design of this work and performed equally. All authors read and approved the final manuscript.

\section{Author details}

${ }^{1}$ Department of Mathematics, King Abdulaziz University, P.O. Box 80203, Jeddah, 21589, Saudi Arabia. ${ }^{2}$ Department of Mathematical Sciences, University of Texas at El Paso, El Paso, Texas, USA. ${ }^{3}$ Department of Mathematics and Statistics, King Fahd University of Petroleum and Minerals, Dhahran, 31261, Saudi Arabia.

Received: 2 June 2013 Accepted: 12 August 2013 Published: 28 August 2013

\section{References}

1. Chistyakov, W: Modular metric spaces, I: basic concepts. Nonlinear Anal. 72(1), 1-14 (2010)

2. Chistyakov, W: Modular metric spaces, Il: application to superposition operators. Nonlinear Anal. 72(1), 15-30 (2010)

3. Nakano, H: Modulared Semi-Ordered Linear Spaces. Maruzen, Tokyo (1950)

4. Musielak, J: Orlicz Spaces and Modular Spaces. Lecture Notes in Math., vol. 1034. Springer, Berlin (1983)

5. Orlicz, W: Collected Papers, Parts I, II. PWN-Polish Scientific Publishers, Warsaw (1988)

6. Diening, L: Theoretical and numerical results for electrorheological fluids. PhD thesis, University of Freiburg, Germany (2002)

7. Ruzicka, M: Electrorheological Fluids Modeling and Mathematical Theory. Springer, Berlin (2002)

8. Harjulehto, P, Hästö, P, Koskenoja, M, Varonen, S: The Dirichlet energy integral and variable exponent Sobolev spaces with zero boundary values. Potential Anal. 25(3), 205-222 (2006)

9. Heinonen, J, Kilpeläinen, T, Martio, O: Nonlinear Potential Theory of Degenerate Elliptic Equations. Oxford University Press, Oxford (1993)

10. Khamsi, MA, Kozlowski, WK, Reich, S: Fixed point theory in modular function spaces. Nonlinear Anal. 14, 935-953 (1990)

11. Khamsi, MA, Kozlowski, WK: On asymptotic pointwise nonexpansive mappings in modular function spaces. J. Math. Anal. Appl. 380, 697-708 (2011)

12. Belluce, LP, Kirk, WA: Nonexpansive mappings and fixed-points in Banach spaces. III. J. Math. 11, 474-479 (1967)

13. Browder, FE: Nonexpansive nonlinear operators in a Banach space. Proc. Natl. Acad. Sci. USA 54, 1041-1044 (1965)

14. Bruck, RE: A common fixed point theorem for a commuting family of nonexpansive mappings. Pac. J. Math. 53, 59-71 (1974)

15. Lim, TC: A fixed point theorem for families of nonexpansive mappings. Pac. J. Math. 53, 487-493 (1974)

16. Goebel, K, Reich, S: Uniform Convexity, Hyperbolic Geometry, and Nonexpansive Mappings. Series of Monographs and Textbooks in Pure and Applied Mathematics, vol. 83. Dekker, New York (1984)

17. Goebel, K, Sekowski, T, Stachura, A: Uniform convexity of the hyperbolic metric and fixed points of holomorphic mappings in the Hilbert ball. Nonlinear Anal. TMA 4, 1011-1021 (1980)

18. Kirk, WA: A fixed point theorem in CAT(0) spaces and $\mathbb{R}$-trees. Fixed Point Theory Appl. 2004(4), $309-316$ (2004)

19. Hussain, N, Khamsi, MA: On asymptotic pointwise contractions in metric spaces. Nonlinear Anal. 71(10), 4423-4429 (2009)

20. Kirk, WA: Fixed points of asymptotic contractions. J. Math. Anal. Appl. 277, 645-650 (2003)

21. Kirk, WA: Asymptotic pointwise contractions. In: 8th International Conference on Fixed Point Theory and Its Applications (Plenary Lecture), Chiang Mai University, Thailand, July 16-22 (2007)

22. Kirk, WA, Xu, H-K: Asymptotic pointwise contractions. Nonlinear Anal. 69, 4706-4712 (2008)

23. Penot, JP: Fixed point theorem without convexity. Analyse non convexity (1977, Pau). Bull. Soc. Math. Fr., Mém. 60 129-152 (1979)

24. Kirk, WA: A fixed point theorem for mappings which do not increase distances. Am. Math. Mon. 72, 1004-1006 (1965)

25. Khamsi, MA: On metric spaces with uniform normal structure. Proc. Am. Math. Soc. 106, 723-726 (1989)

26. Khamsi, MA, Kirk, WA: An Introduction to Metric Spaces and Fixed Point Theory. Wiley, New York (2001)

27. Kozlowski, WM: Modular Function Spaces. Series of Monographs and Textbooks in Pure and Applied Mathematics, vol. 122. Dekker, New York (1988)

28. Kozlowski, WM: Notes on modular function spaces I. Comment. Math. 28, 91-104 (1988) 
29. Kozlowski, WM: Notes on modular function spaces II. Comment. Math. 28, 105-120 (1988)

30. Kirk, WA: An abstract fixed point theorem for nonexpansive mappings. Proc. Am. Math. Soc. 82, 640-642 (1981)

31. Gillespie, AA, Williams, BB: Fixed point theorem for nonexpansive mappings on Banach spaces with uniformly normal structure. Appl. Anal. 9(2), 121-124 (1979)

doi:10.1186/1687-1812-2013-229

Cite this article as: Abdou and Khamsi: On the fixed points of nonexpansive mappings in modular metric spaces.

Fixed Point Theory and Applications 2013 2013:229.

Submit your manuscript to a SpringerOpen ${ }^{\odot}$ journal and benefit from:

- Convenient online submission

- Rigorous peer review

- Immediate publication on acceptance

- Open access: articles freely available online

- High visibility within the field

- Retaining the copyright to your article

Submit your next manuscript at $>$ springeropen.com 